\title{
Insecticide compatibility with abundance and diversity of predatory fauna in wheat (Triticum aestivum) ecosystem
}

Suresh R. Jambagi ( $\sim$ jambagisuru@gmail.com )

University of Agricultural Sciences Dharwad https://orcid.org/0000-0002-4678-8396

D. N. Kambrekar

University of Agricultural Sciences Dharwad

C. P. Mallapur

University of Agricultural Sciences Dharwad

V. Rudra Naik

University of Agricultural Sciences Dharwad

Research Article

Keywords: Abundance, Biocompatibility, Diversity, Insecticides, Natural enemies, Wheat

Posted Date: January 24th, 2022

DOI: https://doi.org/10.21203/rs.3.rs-790293/v1

License: (c) (i) This work is licensed under a Creative Commons Attribution 4.0 International License.

Read Full License 


\section{Abstract}

Assessment of the potential effects of insecticides on the natural enemies is an important part of IPM. The use of natural enemies in combination with selected insecticides which have no effect on them is effective in depressing the population density of the insect pest. In this context, an effort was made to know the diversity of predatory fauna and effect of seed dressers, soil applied chemicals and foliar spraying insecticides on their abundance in wheat during rabi, 2019-20. The investigation registered the activity of green lacewing (Chrysoperla sp.), coccinellids (Coccinella transversalis, Cheilomenes sexmaculata, Illeis cincta), syrphids and spider (Oxyopes sp.). Meanwhile, the results also revealed the peak activity of green lacewing, coccinellids, syrphids and spiders in seed treatment with thiamethoxam 30 FS @ $5 \mathrm{ml} / \mathrm{kg}$ seeds $(1.20,1.40,0.73$ and 0.67 per meter square area, respectively) followed by soil application of carbofuran 3G @ $30 \mathrm{~kg} / \mathrm{ha}(1.17,1.37,0.70$ and 0.62 per meter square, respectively) and both were found on par with untreated check $(1.39,1.51,0.81$ and 0.78 per meter square, respectively). Whereas, the least population was recorded in soil application of carbofuran $3 \mathrm{G} @ 30 \mathrm{~kg} / \mathrm{ha}$ followed by a foliar application of cypermethrin 10 EC @ $0.5 \mathrm{ml} / \mathrm{l}(0.22,0.24,0.14$ and 0.12 per meter square, respectively) over rest of the treatments. These results endorsed the biocompatibility of seed dressers (Thiamethoxam 30 FS), soil applied chemicals (Carbofuran 3G) and detrimental effects of foliar spraying insecticides (Cypermethrin 10 EC, Emamectin benzoate 5SG and Nimbecidine 1500 ppm) on population dynamics of natural enemy.

\section{Introduction}

Wheat (Triticum spp.) is one of the low input cereal crop, being grown in many parts of the world. Due to its unique baking quality, it has got prime importance as major food grain crop and consider it as 'King of Cereals' since from centuries. Wheat is less attacked by insect pests in field as compared to other food grain crops even though, insect pests and diseases together reported to cause 20 to 37 per cent yield loss (Pimentel 1997). In global perspective, 26 insect pests are reported to damage wheat crop, while 12 species have been reported from Indian Sub-continent which infest from planting to till harvest of the crop (Anon. 2013). Since from many years, farmers are depending on many insecticides to tackle this pest attack. Due to indiscriminate usage of such agrochemicals, we are facing many puzzles in recent days viz., development of resistance in insects, outbreak and resurgence of insect pests, gradual decrease in natural enemy population and frequent detection of insecticide residues in agricultural products. This necessitates the identification of alternative sustainable pest management strategies.

Insect natural enemies provide important ecosystem services by suppressing insect pest population in many agricultural crops. Most of the biological control agents including predators, parasitoids and spiders are naturally occurring agents which provide excellent regulation of many insect pests (Schmidt et al. 2004). Candidate predators and parasitoids for IPM programmes should thus be evaluated for susceptible to the pesticides used to control agricultural pests (Hassan et al. 1987). Assessment of the potential effects of insecticides on the natural enemies is therefore an important part of IPM programme (Holland et al. 2000; El-Wakeil et al. 2013). The use of natural enemies in combination with selected 
insecticides which have no effect on them is effective in depressing the population density of the insect pest. With this background, the present study was aimed to evaluate the potential effect of selected insecticides on diversity and abundance of predatory fauna in wheat.

\section{Materials And Methods}

\section{Preparation of research plot}

The present investigation was carried out during rabi 2019-20 at Main Agricultural Research Station (MARS), University of Agricultural Sciences, Dharwad - India by using wheat cultivar UAS-304 on medium black soil with maize as the previous crop under irrigated condition. The experiment was laid out in Randomised Block Design (RBD) with three replications of nine treatments including untreated check (UTC). The crop was sown with a row spacing of $23 \mathrm{~cm}$ apart in a plot size of $3 \mathrm{~m} \times 2.7 \mathrm{~m}$. All the crop production technologies were adopted as per the package of practice.

\section{Selection of insecticides}

The insecticides used for the control of shoot fly (Atherigona spp.), one of the major potential pest (noticed $>26 \%$ dead heart in wheat by Jambagi 2020) in wheat has been considered for the investigation. This tends to reveal the effect of seed dressers, soil applying chemicals, foliar spraying insecticides and their combinations on natural enemies in field condition. Here, the prophylactic application of insecticides either as seed treatment or soil application has been done at the time of sowing whereas, the other foliar treatments were imposed at 15 Days After Emergence DAE by using knapsack sprayer with $450 \mathrm{I} / \mathrm{ha}$ spray fluid.

\section{Estimation of natural enemies}

Predators viz., green lacewing (grubs and adults), coccinellids (grubs and adults), syrphid fly (grubs and adults) and predatory spider (adults) population were recorded per one square meter area in each treatment at a day before spraying and at five, ten and fifteen days after spraying.

\section{Statistical analysis}

The obtained data were subjected to square root $(\sqrt{\mathrm{x}+0.5)})$ transformation, analysis was done by using RBD design and values were analysed using Duncan's Multiple Range Test (DMRT) (Duncan 1955) in MSTAT software.

\section{Results}

The investigation registered the activity of three predatory insects viz., green lacewing (Chrysoperla sp.), coccinellids (Coccinella transversalis, Cheilomenes sexmaculata, Illeis cincta), syrphids and one predatory spider (Oxyopes sp.). The observations recorded on population of Chrysoperla sp. per square meter area in wheat crop at different intervals are presented in Table 1. The pooled mean of chrysopid 
population at 5, 10 and 15 Days After Spraying (DAS) confirmed that the seed treatment with thiamethoxam $30 \mathrm{FS} @ 5 \mathrm{ml} / \mathrm{kg}$ seed $\left(\mathrm{T}_{1}\right)$ recorded maximum population of 1.20 followed by soil application of carbofuran 3G @ $30 \mathrm{~kg} / \mathrm{ha}\left(\mathrm{T}_{2}\right)(1.17)$ besides untreated check (1.39). Whereas, soil application of carbofuran 3G @ $30 \mathrm{~kg} / \mathrm{ha}$ followed by a spray of cypermethrin 10 EC @ $0.5 \mathrm{ml} / \mathrm{l}$ recorded least density (0.22) of chrysopids and found on par with seed treatment with thiamethoxam 30 FS @ 5 $\mathrm{ml} / \mathrm{l}$ along with a spray of cypermethrin 10 EC @ $0.5 \mathrm{ml} / \mathrm{l}$ (0.25). During the investigation, we have recorded three coccinellid predators viz., Coccinella transversalis, Cheilomenes sexmaculata, and IIleis cincta (fungal predator) in the field. As per the data presented in Table 2, the pooled mean of three intervals in different treatments confirmed that the untreated control (UTC) reported maximum coccinellid population of 1.51 per meter square area, followed by $T_{1}$ (1.40) and $T_{2}$ (1.37). Whereas, the least population was noticed in $T_{8}(0.24)$ which was closely followed by $T_{7}(0.28)$. 
Table 1

Impact of insecticidal treatments on Chrysoperla sp. population in wheat

\begin{tabular}{|c|c|c|c|c|c|c|c|}
\hline \multirow{2}{*}{$\begin{array}{l}\text { Tr. } \\
\text { No. }\end{array}$} & \multirow[t]{2}{*}{ Treatments } & \multirow[t]{2}{*}{ Dosage } & \multicolumn{5}{|c|}{ Number of Chrysoperla/m² } \\
\hline & & & 1 DBS & 5 DAS & 10 DAS & $15 \mathrm{DAS}$ & Mean \\
\hline 1 & $\begin{array}{l}\text { Seed treatment with } \\
\text { Thiamethoxam } 30 \text { FS }\end{array}$ & $5 \mathrm{ml} / \mathrm{kg}$ & $\begin{array}{l}0.89 \\
(1.18)^{a}\end{array}$ & $\begin{array}{l}0.94 \\
(1.20)^{a}\end{array}$ & $\begin{array}{l}1.25 \\
(1.32)^{\mathrm{ab}}\end{array}$ & $\begin{array}{l}1.41 \\
(1.38)^{a b}\end{array}$ & 1.20 \\
\hline 2 & $\begin{array}{l}\text { Soil application of } \\
\text { Carbofuran } 3 G\end{array}$ & $\begin{array}{l}30 \\
\mathrm{~kg} / \mathrm{ha}\end{array}$ & $\begin{array}{l}0.81 \\
(1.14)^{\mathrm{a}}\end{array}$ & $\begin{array}{l}0.92 \\
(1.19)^{\mathrm{a}}\end{array}$ & $\begin{array}{l}1.21 \\
(1.31)^{\mathrm{ab}}\end{array}$ & $\begin{array}{l}1.39 \\
(1.37)^{\mathrm{abc}}\end{array}$ & 1.17 \\
\hline 3 & $\begin{array}{l}T_{1}+\text { One spray of } \\
\text { Nimbecidine } 1500 \text { ppm }\end{array}$ & $\begin{array}{l}5 \mathrm{ml} / \mathrm{kg} \\
+ \\
3 \mathrm{ml} / \mathrm{l}\end{array}$ & $\begin{array}{l}0.83 \\
(1.15)^{\mathrm{a}}\end{array}$ & $\begin{array}{l}0.71 \\
(1.10)^{a b}\end{array}$ & $\begin{array}{l}0.79 \\
(1.14)^{\mathrm{ab}}\end{array}$ & $\begin{array}{l}0.98 \\
(1.22)^{b c d}\end{array}$ & 0.83 \\
\hline 4 & $\begin{array}{l}T_{2}+\text { One spray of } \\
\text { Nimbecidine } 1500 \text { ppm }\end{array}$ & $\begin{array}{l}30 \\
\mathrm{~kg} / \mathrm{ha}+ \\
3 \mathrm{ml} / \mathrm{l}\end{array}$ & $\begin{array}{l}0.78 \\
(1.13)^{\mathrm{a}}\end{array}$ & $\begin{array}{l}0.75 \\
(1.12)^{a b}\end{array}$ & $\begin{array}{l}0.76 \\
(1.12)^{\mathrm{ab}}\end{array}$ & $\begin{array}{l}0.92 \\
(1.19)^{\mathrm{cd}}\end{array}$ & 0.81 \\
\hline 5 & $\begin{array}{l}\mathrm{T}_{1}+\text { One spray of } \\
\text { Emamectin benzoate } 5 \mathrm{SG}\end{array}$ & $\begin{array}{l}5 \mathrm{ml} / \mathrm{kg} \\
+ \\
0.2 \mathrm{~g} / \mathrm{l}\end{array}$ & $\begin{array}{l}0.81 \\
(1.14)^{\mathrm{a}}\end{array}$ & $\begin{array}{l}0.45 \\
(0.97)^{b c}\end{array}$ & $\begin{array}{l}0.51 \\
(1.00)^{a b}\end{array}$ & $\begin{array}{l}0.76 \\
(1.12)^{d}\end{array}$ & 0.57 \\
\hline 6 & $\begin{array}{l}\mathrm{T}_{2}+\text { One spray of } \\
\text { Emamectin benzoate } 5 \mathrm{SG}\end{array}$ & $\begin{array}{l}30 \\
\mathrm{~kg} / \mathrm{ha}+ \\
0.2 \mathrm{~g} / \mathrm{l}\end{array}$ & $\begin{array}{l}0.75 \\
(1.12)^{\mathrm{a}}\end{array}$ & $\begin{array}{l}0.41 \\
(0.95)^{\mathrm{bc}}\end{array}$ & $\begin{array}{l}0.49 \\
(0.99)^{\mathrm{ab}}\end{array}$ & $\begin{array}{l}0.74 \\
(1.11)^{d}\end{array}$ & 0.55 \\
\hline 7 & $\begin{array}{l}\mathrm{T}_{1}+\text { One spray of } \\
\text { Cypermethrin } 10 \mathrm{EC}\end{array}$ & $\begin{array}{l}5 \mathrm{ml} / \mathrm{kg} \\
+ \\
0.5 \mathrm{ml} / \mathrm{l}\end{array}$ & $\begin{array}{l}0.86 \\
(1.17)^{\mathrm{a}}\end{array}$ & $\begin{array}{l}0.23 \\
(0.85)^{\mathrm{c}}\end{array}$ & $\begin{array}{l}0.25 \\
(0.87)^{\mathrm{b}}\end{array}$ & $\begin{array}{l}0.28 \\
(0.88)^{\mathrm{e}}\end{array}$ & 0.25 \\
\hline 8 & $\begin{array}{l}\mathrm{T}_{2}+\text { One spray of } \\
\text { Cypermethrin } 10 \mathrm{EC}\end{array}$ & $\begin{array}{l}30 \\
\mathrm{~kg} / \mathrm{ha}+ \\
0.5 \mathrm{ml} / \mathrm{l}\end{array}$ & $\begin{array}{l}0.79 \\
(1.14)^{\mathrm{a}}\end{array}$ & $\begin{array}{l}0.19 \\
(0.83)^{c}\end{array}$ & $\begin{array}{l}0.22 \\
(0.85)^{\mathrm{b}}\end{array}$ & $\begin{array}{l}0.24 \\
(0.86)^{\mathrm{e}}\end{array}$ & 0.22 \\
\hline 9 & Untreated check (UTC) & - & $\begin{array}{l}0.91 \\
(1.19)^{\mathrm{a}}\end{array}$ & $\begin{array}{l}0.95 \\
(1.20)^{\mathrm{a}}\end{array}$ & $\begin{array}{l}1.54 \\
(1.43)^{\mathrm{a}}\end{array}$ & $\begin{array}{l}1.69 \\
(1.48)^{\mathrm{a}}\end{array}$ & 1.39 \\
\hline & S. Em \pm & & 0.08 & 0.06 & 0.06 & 0.07 & - \\
\hline
\end{tabular}

DBS: Day Before Spraying

DAS: Days After Spraying

NS: Non-Significant 


\begin{tabular}{|c|c|c|c|c|c|c|c|}
\hline \multirow{2}{*}{$\begin{array}{l}\text { Tr. } \\
\text { No. }\end{array}$} & \multirow[t]{2}{*}{ Treatments } & \multirow[t]{2}{*}{ Dosage } & \multicolumn{5}{|c|}{ Number of Chrysoperla/m² } \\
\hline & & & 1 DBS & 5 DAS & 10 DAS & 15 DAS & Mean \\
\hline & CD at $5 \%$ & & NS & 0.18 & 0.18 & 0.20 & - \\
\hline & CV (\%) & & 12.92 & 9.76 & 9.64 & 9.82 & - \\
\hline \multicolumn{8}{|c|}{ DBS: Day Before Spraying } \\
\hline \multicolumn{8}{|c|}{ DAS: Days After Spraying } \\
\hline \multicolumn{8}{|c|}{ NS: Non-Significant } \\
\hline
\end{tabular}

*Figures in parentheses are $\sqrt{ } \mathrm{x}+0.5$ transformed values

Means followed by same alphabet in a column do not differ significantly (0.05) by DMRT 
Table 2

Impact of insecticidal treatments on coccinellid population in wheat

\begin{tabular}{|c|c|c|c|c|c|c|c|}
\hline \multirow{2}{*}{$\begin{array}{l}\text { Tr. } \\
\text { No. }\end{array}$} & \multirow[t]{2}{*}{ Treatments } & \multirow[t]{2}{*}{ Dosage } & \multicolumn{5}{|c|}{ Number of coccinellids $/ \mathrm{m}^{2}$} \\
\hline & & & 1 DBS & 5 DAS & 10 DAS & $\begin{array}{l}15 \\
\text { DAS }\end{array}$ & Mean \\
\hline 1 & $\begin{array}{l}\text { Seed treatment with } \\
\text { Thiamethoxam } 30 \text { FS }\end{array}$ & $5 \mathrm{ml} / \mathrm{kg}$ & $\begin{array}{l}0.98 \\
(1.22)^{\mathrm{a}}\end{array}$ & $\begin{array}{l}0.94 \\
(1.20)^{\mathrm{a}}\end{array}$ & $\begin{array}{l}1.40 \\
(1.38)^{\mathrm{a}}\end{array}$ & $\begin{array}{l}1.86 \\
(1.54)^{\mathrm{a}}\end{array}$ & 1.40 \\
\hline 2 & $\begin{array}{l}\text { Soil application of } \\
\text { Carbofuran } 3 G\end{array}$ & $\begin{array}{l}30 \\
\mathrm{~kg} / \mathrm{ha}\end{array}$ & $\begin{array}{l}0.81 \\
(1.14)^{\mathrm{a}}\end{array}$ & $\begin{array}{l}0.95 \\
(1.20)^{\mathrm{a}}\end{array}$ & $\begin{array}{l}1.36 \\
(1.36)^{a}\end{array}$ & $\begin{array}{l}1.81 \\
(1.52)^{\mathrm{a}}\end{array}$ & 1.37 \\
\hline 3 & $\begin{array}{l}\mathrm{T}_{1}+\text { One spray of } \\
\text { Nimbecidine } 1500 \mathrm{ppm}\end{array}$ & $\begin{array}{l}5 \mathrm{ml} / \mathrm{kg} \\
+ \\
3 \mathrm{ml} / \mathrm{l}\end{array}$ & $\begin{array}{l}0.87 \\
(1.17)^{\mathrm{a}}\end{array}$ & $\begin{array}{l}0.65 \\
(1.07)^{\mathrm{ab}}\end{array}$ & $\begin{array}{l}0.96 \\
(1.21)^{\mathrm{ab}}\end{array}$ & $\begin{array}{l}1.28 \\
(1.33)^{b}\end{array}$ & 0.96 \\
\hline 4 & $\begin{array}{l}\mathrm{T}_{2}+\text { One spray of } \\
\text { Nimbecidine } 1500 \mathrm{ppm}\end{array}$ & $\begin{array}{l}30 \\
\mathrm{~kg} / \mathrm{ha}+ \\
3 \mathrm{ml} / \mathrm{l}\end{array}$ & $\begin{array}{l}0.72 \\
(1.10)^{\mathrm{a}}\end{array}$ & $\begin{array}{l}0.63 \\
(1.06)^{a b}\end{array}$ & $\begin{array}{l}0.94 \\
(1.20)^{a b}\end{array}$ & $\begin{array}{l}1.23 \\
(1.32)^{b}\end{array}$ & 0.93 \\
\hline 5 & $\begin{array}{l}\mathrm{T}_{1}+\text { One spray of Emamectin } \\
\text { benzoate } 5 \mathrm{SG}\end{array}$ & $\begin{array}{l}5 \mathrm{ml} / \mathrm{kg} \\
+ \\
0.2 \mathrm{~g} / \mathrm{l}\end{array}$ & $\begin{array}{l}0.86 \\
(1.17)^{\mathrm{a}}\end{array}$ & $\begin{array}{l}0.34 \\
(0.92)^{b c}\end{array}$ & $\begin{array}{l}0.46 \\
(0.98)^{\mathrm{bc}}\end{array}$ & $\begin{array}{l}0.54 \\
(1.02)^{c}\end{array}$ & 0.45 \\
\hline 6 & $\begin{array}{l}\mathrm{T}_{2}+\text { One spray of Emamectin } \\
\text { benzoate } 5 \mathrm{SG}\end{array}$ & $\begin{array}{l}30 \\
\mathrm{~kg} / \mathrm{ha}+ \\
0.2 \mathrm{~g} / \mathrm{l}\end{array}$ & $\begin{array}{l}0.83 \\
(1.15)^{\mathrm{a}}\end{array}$ & $\begin{array}{l}0.31 \\
(0.90)^{b c}\end{array}$ & $\begin{array}{l}0.43 \\
(0.96)^{b c}\end{array}$ & $\begin{array}{l}0.51 \\
(1.00)^{\mathrm{c}}\end{array}$ & 0.42 \\
\hline 7 & $\begin{array}{l}\mathrm{T}_{1}+\text { One spray of } \\
\text { Cypermethrin } 10 \mathrm{EC}\end{array}$ & $\begin{array}{l}5 \mathrm{ml} / \mathrm{kg} \\
+ \\
0.5 \mathrm{ml} / \mathrm{l}\end{array}$ & $\begin{array}{l}0.81 \\
(1.14)^{\mathrm{a}}\end{array}$ & $\begin{array}{l}0.22 \\
(0.85)^{\mathrm{c}}\end{array}$ & $\begin{array}{l}0.28 \\
(0.88)^{\mathrm{c}}\end{array}$ & $\begin{array}{l}0.33 \\
(0.91)^{\mathrm{c}}\end{array}$ & 0.28 \\
\hline 8 & $\begin{array}{l}\mathrm{T}_{2}+\text { One spray of } \\
\text { Cypermethrin } 10 \mathrm{EC}\end{array}$ & $\begin{array}{l}30 \\
\mathrm{~kg} / \mathrm{ha}+ \\
0.5 \mathrm{ml} / \mathrm{l}\end{array}$ & $\begin{array}{l}0.79 \\
(1.14)^{a}\end{array}$ & $\begin{array}{l}0.19 \\
(0.83)^{\mathrm{c}}\end{array}$ & $\begin{array}{l}0.23 \\
(0.85)^{\mathrm{c}}\end{array}$ & $\begin{array}{l}0.29 \\
(0.89)^{\mathrm{c}}\end{array}$ & 0.24 \\
\hline 9 & Untreated check (UTC) & - & $\begin{array}{l}0.99 \\
(1.22)^{a}\end{array}$ & $\begin{array}{l}0.95 \\
(1.20)^{\mathrm{a}}\end{array}$ & $\begin{array}{l}1.49 \\
(1.41)^{\mathrm{a}}\end{array}$ & $\begin{array}{l}2.10 \\
(1.61)^{\mathrm{a}}\end{array}$ & 1.51 \\
\hline & S. Emt & & 0.07 & 0.06 & 0.08 & 0.07 & - \\
\hline
\end{tabular}

DBS: Day Before Spraying

DAS: Days After Spraying

NS: Non-Significant 


\begin{tabular}{|c|c|c|c|c|c|c|c|}
\hline \multirow{2}{*}{$\begin{array}{l}\text { Tr. } \\
\text { No. }\end{array}$} & \multirow[t]{2}{*}{ Treatments } & \multirow[t]{2}{*}{ Dosage } & \multicolumn{5}{|c|}{ Number of coccinellids $/ \mathrm{m}^{2}$} \\
\hline & & & 1 DBS & 5 DAS & 10 DAS & $\begin{array}{l}15 \\
\text { DAS }\end{array}$ & Mean \\
\hline & CD at $5 \%$ & & NS & 0.19 & 0.23 & 0.20 & - \\
\hline & CV (\%) & & 10.27 & 10.70 & 11.90 & 9.63 & - \\
\hline \multicolumn{8}{|c|}{ DBS: Day Before Spraying } \\
\hline \multicolumn{8}{|c|}{ DAS: Days After Spraying } \\
\hline NS: & n-Significant & & & & & & \\
\hline
\end{tabular}

*Figures in parentheses are $\sqrt{x}+0.5$ transformed values

Means followed by same alphabet in a column do not differ significantly (0.05) by DMRT

The similar trend was also observed in abundance of syrphid population (Table 3) where, the pooled mean population of 5,10 and 15 DAS ranged from 0.14 to 0.81 per meter square area with $T_{1}(0.73)$ being superior over other insecticidal treatments besides untreated check $(0.81)$ and closely followed by $T_{2}(0.70)$. During the experiment, we have also looked over the diversity of spider population and registered one predatory spider, Oxyopes sp. in the experimental plots. The pooled mean of three intervals in different treatments also confirmed the trend, where the peak spider population was recorded in $\mathrm{T}_{1}$ (0.67) and $\mathrm{T}_{2}(0.62)$ where they found numerically on par with untreated check (0.78). The lowest density of 0.12 observed from $\mathrm{T}_{8}$ (Table 4 ). In overall observations, the population of all the predators were present to a greater extent within untreated plot compared with the treated ones. 
Table 3

Impact of insecticidal treatments on syrphid population in wheat

\begin{tabular}{|c|c|c|c|c|c|c|c|}
\hline \multirow{2}{*}{$\begin{array}{l}\text { Tr. } \\
\text { No. }\end{array}$} & \multirow[t]{2}{*}{ Treatments } & \multirow[t]{2}{*}{ Dosage } & \multicolumn{5}{|c|}{ Number of syrphids $/ \mathrm{m}^{2}$} \\
\hline & & & 1 DBS & 5 DAS & 10 DAS & 15 DAS & Mean \\
\hline 1 & $\begin{array}{l}\text { Seed treatment with } \\
\text { Thiamethoxam } 30 \mathrm{FS}\end{array}$ & $5 \mathrm{ml} / \mathrm{kg}$ & $\begin{array}{l}0.51 \\
(1.00)^{\mathrm{a}}\end{array}$ & $\begin{array}{l}0.58 \\
(1.04)^{\mathrm{ab}}\end{array}$ & $\begin{array}{l}0.75 \\
(1.12)^{\mathrm{a}}\end{array}$ & $\begin{array}{l}0.86 \\
(1.17)^{\mathrm{ab}}\end{array}$ & 0.73 \\
\hline 2 & $\begin{array}{l}\text { Soil application of } \\
\text { Carbofuran } 3 G\end{array}$ & $\begin{array}{l}30 \\
\mathrm{~kg} / \mathrm{ha}\end{array}$ & $\begin{array}{l}0.43 \\
(0.96)^{\mathrm{a}}\end{array}$ & $\begin{array}{l}0.56 \\
(1.03)^{\mathrm{ab}}\end{array}$ & $\begin{array}{l}0.71 \\
(1.10)^{\mathrm{a}}\end{array}$ & $\begin{array}{l}0.83 \\
(1.15)^{\mathrm{abc}}\end{array}$ & 0.70 \\
\hline 3 & $\begin{array}{l}T_{1}+\text { One spray of } \\
\text { Nimbecidine } 1500 \text { ppm }\end{array}$ & $\begin{array}{l}5 \mathrm{ml} / \mathrm{kg} \\
+ \\
3 \mathrm{ml} / \mathrm{l}\end{array}$ & $\begin{array}{l}0.48 \\
(0.99)^{a}\end{array}$ & $\begin{array}{l}0.42 \\
(0.96)^{\mathrm{abc}}\end{array}$ & $\begin{array}{l}0.62 \\
(1.06)^{\mathrm{ab}}\end{array}$ & $\begin{array}{l}0.66 \\
(1.08)^{a b c}\end{array}$ & 0.57 \\
\hline 4 & $\begin{array}{l}T_{2}+\text { One spray of } \\
\text { Nimbecidine } 1500 \text { ppm }\end{array}$ & $\begin{array}{l}30 \\
\mathrm{~kg} / \mathrm{ha}+ \\
3 \mathrm{ml} / \mathrm{l}\end{array}$ & $\begin{array}{l}0.43 \\
(0.96)^{a}\end{array}$ & $\begin{array}{l}0.40 \\
(0.95)^{\mathrm{abc}}\end{array}$ & $\begin{array}{l}0.63 \\
(1.06)^{a b}\end{array}$ & $\begin{array}{l}0.64 \\
(1.07)^{\mathrm{abc}}\end{array}$ & 0.56 \\
\hline 5 & $\begin{array}{l}\mathrm{T}_{1}+\text { One spray of } \\
\text { Emamectin benzoate } 5 \mathrm{SG}\end{array}$ & $\begin{array}{l}5 \mathrm{ml} / \mathrm{kg} \\
+ \\
0.2 \mathrm{~g} / \mathrm{l}\end{array}$ & $\begin{array}{l}0.49 \\
(0.99)^{\mathrm{a}}\end{array}$ & $\begin{array}{l}0.25 \\
(0.87)^{a b c}\end{array}$ & $\begin{array}{l}0.32 \\
(0.91)^{\mathrm{bc}}\end{array}$ & $\begin{array}{l}0.36 \\
(0.93)^{\mathrm{abc}}\end{array}$ & 0.31 \\
\hline 6 & $\begin{array}{l}\mathrm{T}_{2}+\text { One spray of } \\
\text { Emamectin benzoate } 5 \mathrm{SG}\end{array}$ & $\begin{array}{l}30 \\
\mathrm{~kg} / \mathrm{ha}+ \\
0.2 \mathrm{~g} / \mathrm{l}\end{array}$ & $\begin{array}{l}0.45 \\
(0.97)^{\mathrm{a}}\end{array}$ & $\begin{array}{l}0.24 \\
(0.86)^{\mathrm{bc}}\end{array}$ & $\begin{array}{l}0.29 \\
(0.89)^{b c}\end{array}$ & $\begin{array}{l}0.32 \\
(0.91)^{\mathrm{abc}}\end{array}$ & 0.28 \\
\hline 7 & $\begin{array}{l}\mathrm{T}_{1}+\text { One spray of } \\
\text { Cypermethrin } 10 \mathrm{EC}\end{array}$ & $\begin{array}{l}5 \mathrm{ml} / \mathrm{kg} \\
+ \\
0.5 \mathrm{ml} / \mathrm{l}\end{array}$ & $\begin{array}{l}0.49 \\
(0.99)^{a}\end{array}$ & $\begin{array}{l}0.11 \\
(0.78)^{\mathrm{c}}\end{array}$ & $\begin{array}{l}0.18 \\
(0.82)^{\mathrm{c}}\end{array}$ & $\begin{array}{l}0.21 \\
(0.84)^{\mathrm{bc}}\end{array}$ & 0.17 \\
\hline 8 & $\begin{array}{l}\mathrm{T}_{2}+\text { One spray of } \\
\text { Cypermethrin } 10 \mathrm{EC}\end{array}$ & $\begin{array}{l}30 \\
\mathrm{~kg} / \mathrm{ha}+ \\
0.5 \mathrm{ml} / \mathrm{l}\end{array}$ & $\begin{array}{l}0.42 \\
(0.96)^{\mathrm{a}}\end{array}$ & $\begin{array}{l}0.09 \\
(0.77)^{\mathrm{c}}\end{array}$ & $\begin{array}{l}0.15 \\
(0.81)^{\mathrm{c}}\end{array}$ & $\begin{array}{l}0.17 \\
(0.82)^{\mathrm{c}}\end{array}$ & 0.14 \\
\hline 9 & Untreated check (UTC) & - & $\begin{array}{l}0.52 \\
(1.01)^{a}\end{array}$ & $\begin{array}{l}0.62 \\
(1.06)^{\mathrm{a}}\end{array}$ & $\begin{array}{l}0.79 \\
(1.14)^{\mathrm{a}}\end{array}$ & $\begin{array}{l}1.01 \\
(1.23)^{\mathrm{a}}\end{array}$ & 0.81 \\
\hline & S. Emt & & 0.06 & 0.07 & 0.07 & 0.09 & - \\
\hline
\end{tabular}

DBS: Day Before Spraying

DAS: Days After Spraying

NS: Non-Significant 


\begin{tabular}{|c|c|c|c|c|c|c|c|}
\hline \multirow{2}{*}{$\begin{array}{l}\text { Tr. } \\
\text { No. }\end{array}$} & \multirow[t]{2}{*}{ Treatments } & \multirow[t]{2}{*}{ Dosage } & \multicolumn{5}{|c|}{ Number of syrphids $/ \mathrm{m}^{2}$} \\
\hline & & & 1 DBS & 5 DAS & 10 DAS & 15 DAS & Mean \\
\hline & CD at $5 \%$ & & NS & 0.21 & 0.23 & 0.28 & - \\
\hline & CV (\%) & & 9.37 & 10.49 & 10.64 & 12.92 & - \\
\hline \multicolumn{8}{|c|}{ DBS: Day Before Spraying } \\
\hline \multicolumn{8}{|c|}{ DAS: Days After Spraying } \\
\hline \multicolumn{8}{|c|}{ NS: Non-Significant } \\
\hline
\end{tabular}

*Figures in parentheses are $\sqrt{ } \mathrm{x}+0.5$ transformed values

Means followed by same alphabet in a column do not differ significantly (0.05) by DMRT 
Table 4

Impact of insecticidal treatments on spider population in wheat

\begin{tabular}{|c|c|c|c|c|c|c|c|}
\hline \multirow{2}{*}{$\begin{array}{l}\text { Tr. } \\
\text { No. }\end{array}$} & \multirow[t]{2}{*}{ Treatments } & \multirow[t]{2}{*}{ Dosage } & \multicolumn{5}{|c|}{ Number of spiders $/ \mathrm{m}^{2}$} \\
\hline & & & 1 DBS & 5 DAS & $10 \mathrm{DAS}$ & $15 \mathrm{DAS}$ & Mean \\
\hline 1 & $\begin{array}{l}\text { Seed treatment with } \\
\text { Thiamethoxam } 30 \text { FS }\end{array}$ & $5 \mathrm{ml} / \mathrm{kg}$ & $\begin{array}{l}0.37 \\
(0.93)^{\mathrm{a}}\end{array}$ & $\begin{array}{l}0.51 \\
(1.00)^{\mathrm{ab}}\end{array}$ & $\begin{array}{l}0.58 \\
(1.04)^{\mathrm{ab}}\end{array}$ & $\begin{array}{l}0.92 \\
(1.19)^{\mathrm{ab}}\end{array}$ & 0.67 \\
\hline 2 & $\begin{array}{l}\text { Soil application of } \\
\text { Carbofuran } 3 G\end{array}$ & $\begin{array}{l}30 \\
\mathrm{~kg} / \mathrm{ha}\end{array}$ & $\begin{array}{l}0.28 \\
(0.88)^{a}\end{array}$ & $\begin{array}{l}0.48 \\
(0.99)^{\mathrm{ab}}\end{array}$ & $\begin{array}{l}0.51 \\
(1.00)^{\mathrm{abc}}\end{array}$ & $\begin{array}{l}0.87 \\
(1.17)^{\mathrm{abc}}\end{array}$ & 0.62 \\
\hline 3 & $\begin{array}{l}T_{1}+\text { One spray of } \\
\text { Nimbecidine } 1500 \text { ppm }\end{array}$ & $\begin{array}{l}5 \mathrm{ml} / \mathrm{kg} \\
+ \\
3 \mathrm{ml} / \mathrm{l}\end{array}$ & $\begin{array}{l}0.40 \\
(0.95)^{\mathrm{a}}\end{array}$ & $\begin{array}{l}0.26 \\
(0.87)^{a b c}\end{array}$ & $\begin{array}{l}0.29 \\
(0.89)^{b c d}\end{array}$ & $\begin{array}{l}0.54 \\
(1.02)^{b c d}\end{array}$ & 0.36 \\
\hline 4 & $\begin{array}{l}T_{2}+\text { One spray of } \\
\text { Nimbecidine } 1500 \text { ppm }\end{array}$ & $\begin{array}{l}30 \\
\mathrm{~kg} / \mathrm{ha}+ \\
3 \mathrm{ml} / \mathrm{l}\end{array}$ & $\begin{array}{l}0.34 \\
(0.92)^{\mathrm{a}}\end{array}$ & $\begin{array}{l}0.24 \\
(0.86)^{\mathrm{abc}}\end{array}$ & $\begin{array}{l}0.28 \\
(0.88)^{\mathrm{bcd}}\end{array}$ & $\begin{array}{l}0.51 \\
(1.00)^{\mathrm{cd}}\end{array}$ & 0.34 \\
\hline 5 & $\begin{array}{l}\mathrm{T}_{1}+\text { One spray of } \\
\text { Emamectin benzoate } 5 \mathrm{SG}\end{array}$ & $\begin{array}{l}5 \mathrm{ml} / \mathrm{kg} \\
+ \\
0.2 \mathrm{~g} / \mathrm{l}\end{array}$ & $\begin{array}{l}0.45 \\
(0.97)^{\mathrm{a}}\end{array}$ & $\begin{array}{l}0.19 \\
(0.83)^{b c}\end{array}$ & $\begin{array}{l}0.21 \\
(0.84)^{\mathrm{cd}}\end{array}$ & $\begin{array}{l}0.32 \\
(0.91)^{\mathrm{de}}\end{array}$ & 0.24 \\
\hline 6 & $\begin{array}{l}\mathrm{T}_{2}+\text { One spray of } \\
\text { Emamectin benzoate } 5 \mathrm{SG}\end{array}$ & $\begin{array}{l}30 \\
\mathrm{~kg} / \mathrm{ha}+ \\
0.2 \mathrm{~g} / \mathrm{l}\end{array}$ & $\begin{array}{l}0.37 \\
(0.93)^{a}\end{array}$ & $\begin{array}{l}0.17 \\
(0.82)^{\mathrm{bc}}\end{array}$ & $\begin{array}{l}0.19 \\
(0.83)^{\mathrm{cd}}\end{array}$ & $\begin{array}{l}0.28 \\
(0.88)^{\mathrm{de}}\end{array}$ & 0.21 \\
\hline 7 & $\begin{array}{l}\mathrm{T}_{1}+\text { One spray of } \\
\text { Cypermethrin } 10 \mathrm{EC}\end{array}$ & $\begin{array}{l}5 \mathrm{ml} / \mathrm{kg} \\
+ \\
0.5 \mathrm{ml} / \mathrm{l}\end{array}$ & $\begin{array}{l}0.44 \\
(0.97)^{\mathrm{a}}\end{array}$ & $\begin{array}{l}0.10 \\
(0.77)^{\mathrm{c}}\end{array}$ & $\begin{array}{l}0.14 \\
(0.80)^{d}\end{array}$ & $\begin{array}{l}0.15 \\
(0.81)^{\mathrm{e}}\end{array}$ & 0.13 \\
\hline 8 & $\begin{array}{l}\mathrm{T}_{2}+\text { One spray of } \\
\text { Cypermethrin } 10 \mathrm{EC}\end{array}$ & $\begin{array}{l}30 \\
\mathrm{~kg} / \mathrm{ha}+ \\
0.5 \mathrm{ml} / \mathrm{l}\end{array}$ & $\begin{array}{l}0.29 \\
(0.89)^{a}\end{array}$ & $\begin{array}{l}0.10 \\
(0.77)^{\mathrm{c}}\end{array}$ & $\begin{array}{l}0.12 \\
(0.79)^{d}\end{array}$ & $\begin{array}{l}0.13 \\
(0.79)^{\mathrm{e}}\end{array}$ & 0.12 \\
\hline 9 & Untreated check (UTC) & - & $\begin{array}{l}0.46 \\
(0.98)^{\mathrm{a}}\end{array}$ & $\begin{array}{l}0.56 \\
(1.03)^{\mathrm{a}}\end{array}$ & $\begin{array}{l}0.75 \\
(1.12)^{\mathrm{a}}\end{array}$ & $\begin{array}{l}1.02 \\
(1.23)^{\mathrm{a}}\end{array}$ & 0.78 \\
\hline & S. Emt & & 0.06 & 0.06 & 0.06 & 0.06 & - \\
\hline
\end{tabular}

DBS: Day Before Spraying

DAS: Days After Spraying

NS: Non-Significant 


\begin{tabular}{|c|c|c|c|c|c|c|c|}
\hline \multirow{2}{*}{$\begin{array}{l}\text { Tr. } \\
\text { No. }\end{array}$} & \multirow[t]{2}{*}{ Treatments } & \multirow[t]{2}{*}{ Dosage } & \multicolumn{5}{|c|}{ Number of spiders $/ \mathrm{m}^{2}$} \\
\hline & & & 1 DBS & 5 DAS & 10 DAS & 15 DAS & Mean \\
\hline & CD at $5 \%$ & & NS & 0.19 & 0.17 & 0.18 & - \\
\hline & CV (\%) & & 11.68 & 12.51 & 11.01 & 10.17 & - \\
\hline \multicolumn{8}{|c|}{ DBS: Day Before Spraying } \\
\hline \multicolumn{8}{|c|}{ DAS: Days After Spraying } \\
\hline NS: & on-Significant & & & & & & \\
\hline
\end{tabular}

*Figures in parentheses are $\sqrt{ } \mathrm{x}+0.5$ transformed values

Means followed by same alphabet in a column do not differ significantly (0.05) by DMRT

\section{Discussion}

A comprehensive understanding of biological and environmental interaction in agriculture, which constitutes a phytobiome is an important focal point for the development of sustainable crop production approach (Way and Heong 1994). This could advocate us regarding the soil health maintenance and natural tackling of many insect pests in the field condition. In the modern agriculture, utilisation of many agrochemicals including fungicides, herbicides, insecticides etc. can hit ecosystem badly with huge deleterious effects, as a result we are facing many health issues in recent days. Therefore, it is clearly evident that the new approaches towards modernising agriculture are must needed and to this end, there must be fundamental knowledge to estimate the required levels of biodiversity in agricultural crops. There are several natural agents are recorded in wheat field, which have potential to take care of many of insect pests in the field. The most diverse group of predators viz., Coleoptera (Coccinellidae), Diptera (Syrphidae), Neuroptera (Chrysopidae and Hemerobidae), Aranea and many hymenopteran wasps were denoted in the arthropoda faunal structure as reported by Malschi (2008) who pioneer in scientific studies on insect pest of wheat and their associated natural enemies and conducted extensive research in winter wheat for several years. Meanwhile, National Institute of Plant Health Management (NIPHM)Government of India also enlisted several predatory insects in AESA based IPM Package for wheat (Anonymous, 2014). So, in this context the present investigation emphasises the biosafety of chemical insecticides to the predatory fauna in field condition.

In this extensive investigation, there is no significant difference among the treatments including untreated check in the natural enemy population (green lace wing, coccinellids, syrphids and spiders) a day before spraying, though they received chemicals at the time of sowing either as seed treatment or soil application. The present findings can be supported by Anita (2009) who observed Chrysoperla sp. and coccinellid population per plant in imidacloprid 70 WS ( $2 \mathrm{~g} / \mathrm{kg}$ seed) and endosulfan $35 \mathrm{EC}(0.07 \%)$ treated plots were on par with untreated check. Biradar (2015) also confirmed the similar trend of 
chrysoperla and coccinellid population dynamics in seed dressers, who noticed non-significant difference among the chemical treatments including untreated control. As the prophylactic seed dressing chemicals not directly come in contact with natural enemies, there will be least direct harmful effect and they remain safe to predatory fauna. The observations recorded at different intervals, after the insecticide spray endorsed the significant difference among the treatments regarding predatory insects. The peak activity of all the natural enemies (green lacewing, coccinellids, syrphids and spiders) was observed in $T_{1}$ (seed treatment with thiamethoxam $30 \mathrm{FS} @ 5 \mathrm{ml} / \mathrm{kg}$ seed) and $\mathrm{T}_{2}$ (soil application of carbofuran $3 \mathrm{G} @ 30$ $\mathrm{kg} / \mathrm{ha}$ ) which were on par with untreated check. Whereas, the least population was recorded in $T_{8}$ and $T_{7}$ where the foliar spray of cypermethrin $10 \mathrm{EC}(0.5 \mathrm{ml} / \mathrm{l})$ was found to be having some detrimental effects on predators. Croft and Whalon (1982) and Brown et al. (1990) studied the effect of some pyrethroid insecticides on natural enemies of some agricultural crops and noticed similar results as of us. Pyrethroid insecticides caused decline in coccinellid population to the extent of 38 to 72 per cent (Meena et al. 2002; Solangi et al. 2007) which is in agreement with the present findings. The similar detrimental effects were also observed in many coccinellid predators (Smith and Stratton 1986; Duffield and Aebischer 1994; Wiles and Jepson 1994; Holland et al. 2000; Meena et al. 2002) and syrphids (Swaran 1999). Earlier workers investigated the effect of neem products on green lacewing (Kaethner 1991; Srinivas and Babu 2000; El-Wakeil et al. 2006), predatory spider (Thomas et al. 1990; Dinter 1995; Schmutterer 1997) and found that, all the neem based botanical insecticides were safer to them. Meanwhile, Katole and Patil (2000), Ahire (2008) and Prakash (2014) noticed higher population of coccinellids, chrysopids and spiders in thiamethoxam $30 \mathrm{FS}$ ( $5 \mathrm{ml} / \mathrm{kg}$ seed) seed treated sorghum plots followed by imidacloprid 17.8 $\mathrm{SL}(10 \mathrm{ml} / \mathrm{kg}$ seed). Balasaheb (2014) reported peak population of coccinellids and Crossopalpus sp. in seed treated with imidacloprid 48 FS followed by thiamethoxam 25 WG in sorghum, which highlights the benefits of prophylactic chemical application.

\section{Conclusions}

From the foregone discussion, it is evident that the seed dressing and soil applied chemicals were relatively safer and can be incorporated in future pest management programmes, where the natural biocontrol agents play a vital role. Nevertheless, our investigation also substantiated the apparent deleterious qualitative and quantitative effects of foliar spraying insecticides on natural enemy population. In this context, there should be consideration of extent of activity and potential of natural predatory and parasitoid insects while recommending the insecticide spray.

\section{Declarations}

Acknowledgements

Not applicable

\section{Funding}


Not applicable

\section{Competing interests}

The authors declare that they have no competing interests

\section{Availability of data and materials}

All data generated during this study are included in this manuscript and available with corresponding author

\section{Code availability}

Not applicable

\section{Authors' contributions}

All the authors were contributed to the study where, first author was Post Graduate student who conducted the experiment while, second author was advisory committee chairman and rest of the two authors were the members of advisory committee of the student and actively involved in analysis and manuscript preparation. All authors read and approved the final manuscript.

\section{Ethics approval and consent to participate}

Not applicable

\section{Consent to participate}

Not applicable

\section{Consent for publication}

Not applicable

\section{References}

1. Ahire RV (2008) Evaluation of newer insecticide thiamethoxam as seed dresser for the management of early season pests in soybean. Doctoral dissertation, Vasantrao Naik Krishi Vidyapeeth, Parbhani

2. Anita VS (2009) Non-chemical approaches for the management of shoot fly Atherigona soccata Rondani in kharif sorghum. Dissertation, University of Agricultural Sciences Dharwad, Karnataka, India

3. Anonymous (2013) Hundred years wheat research in India. Directorate of Wheat Research, Karnal, pp 171-172 
4. Anonymous (2014) Satyagopal K, Sushil SN, Jeyakumar P, Shankar G, Sharma OP, Sain SK, Boina DR, Chattopadhyay D, Srinivasa Rao N, Sunanda BS, Asre R, Murali R, Kapoor KS, Arya S, Kumar S, Patni CS, Sharma I, Ravindra H, Shivanna BK, Saharan MS, Kaur D, Singh B, Sharma RK, Chokar RS, Karnatak AK, Ruchira Tiwari, Deepshikha, BR Patel, Surajit Khalko, Nripendra Laskar, Ayon Roy, Tapan Kumar Hath, LV Ghetiya, Sathyanarayana N, Latha S (2014) AESA based IPM package for Wheat. pp 58. https://farmer.gov.in/imagedefault/ipm/wheat.pdf

5. Balasaheb BA (2014) Bio efficacy of different insecticides against sorghum shoot fly, Atherigona soccata Rondani in Rabi season. Dissertation, MPKV, Rahuri, Maharashtra, India

6. Biradar A (2015) Management of shoot fly (Atherigona soccata Rondani) in Rabi sorghum. Dissertation, University of Agricultural Sciences Dharwad, Karnataka, India

7. Brown GW, Bifulco A, Veiel HOF, Andrews B (1990) Self-esteem and depression. Social psych psych epidem 25(5):225-234

8. Croft BA, Whalon ME (1982) Selective toxicity of pyrethroid insecticides to arthropod natural enemies and pests of agricultural crops. Entomophaga 27(1):3-21

9. Dinter A (1995) Untersuchungen zur Populationsdynamik von Spinnen (Arachnida: Araneae) in Winterweizen und deren Beeinflussung durch insektizide Wirkstoffe [dissertation]. [Göttingen]: Cuvillier Publisher, Goettingen Universität. ISBN 3-89588-171-6 Cuvillier Verlag Göttingen 383 p

10. Duffield SJ, Aebischer NJ (1994) The effect of spatial scale of treatment with dimethoate on invertebrate population recovery in winter wheat. J Appl Ecol 31:263-281

11. Duncan DB (1955) Multiple range and multiple $F$ tests. Biometrics 11(1):1-42

12. El-Wakeil NE, Gaafar N, Vidal S (2006) Side effect of some Neem products on natural enemies of Helicoverpa, Trichogramma spp. and Chrysoperla carnea. Arch Phytopathol Plant Prot 39:445-455

13. El-Wakeil N, Gaafar N, Sallam A, Volkmar C (2013) Side effects of insecticides on natural enemies and possibility of their integration in plant protection strategies. In: Trdan S (ed) Development of Safer and More Effective Technologies Agricultural and Biological Sciences. InTech Open Access Publisher, Insecticides, pp 1-56

14. Hassan SA, Albert R, Bigler F, Blaisinger P, Bogenschütz H, Boller E, Vanwetswinkel G (1987) Results of the third joint pesticide testing programme by the IOBC/WPRS-Working Group "Pesticides and Beneficial Organisms". J Appl Entomol 103(1-5):92-107

15. Holland JM, Winder L, Perry JN (2000) The impact of dimethoate on the spatial distribution of beneficial arthropods in winter wheat. Ann appl biol 136(2):93-105

16. Jambagi SR (2020) Status and management of shoot fly in wheat. Dissertation, University of Agricultural Sciences Dharwad, Karnataka, India

17. Kaethner M (1991) No side effects of neem on the aphidophagous predators Chrysoperla carnea and Coccinella septempunctata. Anz Schädlngskde Planzenschutz Umweltschutz 64:97-99

18. Katole SR, Patil PJ (2000) Biosafety of imidacloprid and thiamethoxam as seed treatment and foliar sprays to some predators. Pestology 24(11):11-13 
19. Malschi D (2008) Environment, agriculture, sustainable development. Optimization of integrated wheat pest management technologies under the dynamics of agroecological changes in Transylvania. Ed. Argonaut, Cluj-Napoca, 250

20. Meena BL, Dadhich SR, Kumawat RL (2002) Efficacy of some insecticides against ladybird beetle, Coccinella septumpunctata L. feeding on fenugreek aphid, Acyrthosiphon pisum (Harris.). Ann Biol 18:171-173

21. Pimentel $D$ (1997) Techniques for reducing pesticide use: economic and environmental benefits. John Wiley and Sons

22. Prakash KS (2014) Efficacy of different seed dressers on sorghum shoot fly Atherigona soccata (Rondani). Dissertation, University of Agricultural Sciences Dharwad, Karnataka, India

23. Schmidt MH, Thewes U, Thies C, Tscharntke T (2004) Aphid suppression by natural enemies in mulched cereals. Entomol Experiment et Applicata 113(2):87-93

24. Schmutterer $\mathrm{H}$ (1997) Side effects of neem (Azadirachta indica) products on insect pathogens and natural enemies of spider mites and insects. J Appl Entomol 121:121-128

25. Smith TM, Stratton GW (1986) Effects of synthetic pyrethroid insecticides on non-target organisms. Residue Rev 97:93-120

26. Solangi BK, Lanjar AG, Lohar MK (2007) Comparative toxicity of some insecticides on 4th instar grub of Coccinella septempunctata L. under laboratory conditions. Sarhad J Agric 23:1091-1096

27. Srinivasan G, Babu PCS (2000) Effect of neem products on predatory green lacewing, Chrysoperla carnea. Pest Res J 12:123-126

28. Swaran D (1999) Effect of some important insecticides on the adults of Coccinella septempunctata L. Predating on different aphid species. J Entomol Res 23:127-131

29. Thomas CFG, Hol EHA, Everts JW (1990) Modelling the diffusion component of dispersal during recovery of a population of linyphiid spiders from exposure to an insecticide. Func Ecolo 4:357-368

30. Way MJ, Heong KL (1994) The role of biodiversity in the dynamics and management of insect pests of tropical irrigated rice-a review. Bull Entomol Res 84(4):567-587

31. Wiles JA, Jepson PC (1994) Sub-lethal effects of deltamethrin residues on the within-crop behaviour and distribution of Coccinella septempunctata. Entomol Exp Appl 72:33-45 